VOL. 21, No. 1 (2017), 87-94

\title{
A generalization of modules with the property $\left(P^{*}\right)$
}

\author{
BuRCU NişANCI TÜRKMEN
}

\begin{abstract}
I.A- Khazzi and P.F. Smith called a module $M$ have the property $\left(P^{*}\right)$ if every submodule $N$ of $M$ there exists a direct summand $K$ of $M$ such that $K \leq N$ and $\frac{N}{K} \subseteq \operatorname{Rad}\left(\frac{M}{K}\right)$. Motivated by this, it is natural to introduce another notion that we called modules that have the properties $\left(G P^{*}\right)$ and $\left(N-G P^{*}\right)$ as proper generalizations of modules that have the property $\left(P^{*}\right)$. In this paper we obtain various properties of modules that have properties $\left(G P^{*}\right)$ and $\left(N-G P^{*}\right)$. We show that the class of modules for which every direct summand is a fully invariant submodule that have the property $\left(G P^{*}\right)$ is closed under finite direct sums. We completely determine the structure of these modules over generalized f-semiperfect rings.
\end{abstract}

\section{INTRODUCTION}

Throughout this paper, all rings are associative with identity element and all modules are unital right $R$-modules. Let $R$ be a ring and let $M$ be an $R$-module. The notation $N \leq M$ means that $N$ is a submodule of $M$. A module $M$ is called extending if every submodule of $M$ is essential in a direct summand of $M$ [4]. Here a submodule $L \leq M$ is said to be essential in $M$, denoted as $L \unlhd M$, if $L \cap N \neq 0$ for every nonzero submodule $N \leq M$. Dually, a submodule $S$ of $M$ is called small (in $M$ ), denoted as $S<<M$, if $M \neq S+L$ for every proper submodule $L$ of $M$. By $\operatorname{Rad}(M)$, we denote the intersection of all maximal submodules of $M$. An $R$-module $M$ is called supplemented if every submodule $N$ of $M$ has a supplement, that is a submodule $K$ minimal with respect to $M=N+K$. Equivalently, $M=N+K$ and $N \cap K<<K$ [11]. $M$ is called ( $\left.f_{-}\right)$supplemented if every (finitely generated) submodule of $M$ has a supplement in $M$ (see [11]). On the other hand, $M$ is called amply supplemented if, for any submodules $N$ and $K$ of $M$ with $M=N+K, K$ contains a supplement of $N$ in $M$. Accordingly a module $M$ is called amply f-supplemented if every finitely generated submodule of $M$ satisfies same condition. It is clear that (amply) f-supplemented modules are a proper generalization of (amply) supplemented modules.

2010 Mathematics Subject Classification. 16D10, 16N80.

Key words and phrases. Generalized f-semiperfect ring, the properties $\left(P^{*}\right),\left(G P^{*}\right)$ and $\left(N-G P^{*}\right)$. 
A module $M$ is called lifting if for every submodule $N$ of $M$ there exists a direct summand $K$ of $M$ such that $K \leq N$ and $\frac{N}{K}<<\frac{M}{K}$ (i.e. $K$ is a coessential submodule of $N$ in $M$ ) as a dual notion of extending modules. Mohamed and Müller has generalized the concept of lifting modules to $\oplus$ supplemented modules. $M$ is called $\oplus$-supplemented if every submodule of $M$ has a supplement that is a direct summand of $M$ [6].

Let $M$ be an $R$-module and let $N$ and $K$ be any submodules of $M$. If $M=N+K$ and $N \cap K \subseteq \operatorname{Rad}(K)$, then $K$ is called a Rad-supplement of $N$ in $M$ [12](according to [10], generalized supplement). It is clear that every supplement is Rad-supplement. $M$ is called Rad-supplemented (according to [10], generalized supplemented) if every submodule of $M$ has a Rad-supplement in $M$, and $M$ is called amply Rad-supplemented if, for any submodules $N$ and $K$ of $M$ with $M=N+K, K$ contains a Rad-supplement of $N$ in $M$. An $R$-module $M$ is called $f$-Rad-supplemented if every finitely generated submodule of $M$ has a Rad-supplement in $M$, and a module $M$ is called amply f-Rad-supplemented if every finitely generated submodule of $M$ has ample Rad-supplements in $M$ (see [7]). A module $M$ is called Rad$\oplus$-supplemented if every submodule has a Rad-supplement that is a direct summand of $M[3]$ and [5].

Recall from Al-Khazzi and Smith [1] that a module $M$ is said to have the property $\left(P^{*}\right)$ if for every submodule $N$ of $M$ there exists a direct summand $K$ of $M$ such that $K \leq N$ and $\frac{N}{K} \subseteq \operatorname{Rad}\left(\frac{M}{K}\right)$. The authors have obtained in the same paper the various properties of modules with the property $\left(P^{*}\right)$. Radical modules have the property $\left(P^{*}\right)$. It is clear that every lifting module has the property $\left(P^{*}\right)$ and every module with the property $\left(P^{*}\right)$ is $\operatorname{Rad}-\oplus$ supplemented.

Let $f: P \longrightarrow M$ be an epimorphism. If $\operatorname{Ker}(f)<<P$, then $f$ is called cover, and if $P$ is a projective module, then a cover $f$ is called a projective cover [11]. Xue [12] calls $f$ a generalized cover if $\operatorname{Ker}(f) \leq \operatorname{Rad}(P)$, and calls a generalized cover $f$ a generalized projective cover if $P$ is a projective module. In the spirit of [12], a module $M$ is said to be (generalized) semiperfect if every factor module of $M$ has a (generalized) projective cover. A module $M$ is said to be $f$-semiperfect if, for every finitely generated submodule $U \leq M$, the factor module $\frac{M}{U}$ has a projective cover in $M$ [11]. Let $M$ be an $R$-module. $M$ is called generalized f-semiperfect module if, for every finitely generated submodule $U \leq M$, the factor module $\frac{M}{U}$ has a generalized projective cover in $M$ [8].

In this study, we obtain some elementary facts about the properties $\left(G P^{*}\right)$ and $\left(N-G P^{*}\right)$ which are a proper generalizations of the property $\left(P^{*}\right)$. Especially, we give a relation for $G^{*}$-supplemented modules. We prove that every direct summand of a module that have the property $\left(G P^{*}\right)$ has the property $\left(G P^{*}\right)$. We show that a module $M$ has the property $\left(N-G P^{*}\right)$ if and only if, for all direct summands $M^{\prime}$ and a coclosed submodule $N^{\prime}$ of $N$, 
$M^{\prime}$ has the property $\left(N^{\prime}-G P^{*}\right)$ for right $R$-modules $M$ and $N$. We obtain that Let $M=\oplus_{i=1}^{n} M_{i}$ be a module and $M_{i}$ is a fully invariant submodule of $M$ for all $i \in\{1,2, \ldots, n\}$. Then $M$ has the property $\left(G P^{*}\right)$ if and only if $M_{i}$ has the property $\left(G P^{*}\right)$ for all $i \in\{1,2, \ldots, n\}$. We illustrate a module with the property $\left(G P^{*}\right)$ which doesn't have the property $\left(P^{*}\right)$. We give a characterization of generalized f-semiperfect rings via the property $\left(G P^{*}\right)$.

\section{Modules with the Properties of $\left(G P^{*}\right)$ And $\left(N-G P^{*}\right)$}

Definition 2.1. A module $M$ has the property $\left(G P^{*}\right)$ if, for every $\gamma \in$ $\operatorname{End}_{R}(M)$ there exists a direct summand $N$ of $M$ such that $N \subseteq \operatorname{Im}(\gamma)$ and $\frac{\operatorname{Im} \gamma}{N} \subseteq \operatorname{Rad}\left(\frac{M}{N}\right)$.

Proposition 2.1. The following conditions are equivalent for a module $M$.

(1) $M$ has the property $\left(G P^{*}\right)$.

(2) For every $\gamma \in \operatorname{End}_{R}(M)$, there exists a decomposition $M=M_{1} \oplus M_{2}$ such that $M_{1} \subseteq \operatorname{Im}(\gamma)$ and $M_{2} \cap \operatorname{Im}(\gamma) \subseteq \operatorname{Rad}\left(M_{2}\right)$.

(3) For every $\gamma \in \operatorname{End}_{R}(M), \operatorname{Im}(\gamma)$ can be represented as $\operatorname{Im} \gamma=N \oplus$ $N^{\prime}$, where $N$ is a direct summand of $M$ and $N^{\prime} \subseteq \operatorname{Rad}(M)$.

Proof. (1) $\Rightarrow(2)$ By the hypothesis, there exist direct summands $M_{1}, M_{2}$ of $M$ such that $M_{1} \subseteq \operatorname{Im}(\gamma), M=M_{1} \oplus M_{2}$ and $\frac{\operatorname{Im}(\gamma)}{M_{1}} \subseteq \operatorname{Rad}\left(\frac{M}{M_{1}}\right)$. Since $M_{2}$ is a $R a d$-supplement of $M_{1}$ in $M, \operatorname{Rad}\left(\frac{M}{M_{1}}\right)=\frac{\operatorname{Rad}(M)+M_{1}}{M_{1}}$ (See [13, Lemma 1.1]). Then $\frac{\operatorname{Im}(\gamma)}{M_{1}} \subseteq \frac{\operatorname{Rad}(M)+M_{1}}{M_{1}}$. So we have $\operatorname{Im}(\gamma) \subseteq \operatorname{Rad}\left(M_{2}\right)+M_{1}$. By the modular law, $M_{2} \cap \operatorname{Im}(\gamma) \subseteq \operatorname{Rad}\left(M_{2}\right)$.

$(2) \Rightarrow(3)$ For every $\gamma \in \operatorname{End}_{R}(M)$, there exists a decomposition $M=$ $M_{1} \oplus M_{2}$ such that $M_{1} \subseteq \operatorname{Im}(\gamma)$ and $M_{2} \cap \operatorname{Im}(\gamma) \subseteq \operatorname{Rad}\left(M_{2}\right)$. So $\operatorname{Im}(\gamma)=$ $M_{1} \oplus\left(\operatorname{Im}(\gamma) \cap M_{2}\right)$ by the modular law. Say $N=M_{1}$ and $N^{\prime}=\operatorname{Im}(\gamma) \cap M_{2}$. Therefore $\operatorname{Im}(\gamma)=N \oplus N^{\prime}$, where $N$ is a direct summand of $M$ and $N^{\prime} \subseteq$ $\operatorname{Rad}(M)$.

$(3) \Rightarrow(1)$ By the hypothesis, for every $\gamma \in \operatorname{End}_{R}(M), \operatorname{Im}(\gamma)=N \oplus N^{\prime}$ where $N$ is a direct summand of $M$ and $N^{\prime} \subseteq \operatorname{Rad}(M)$. Thus there exists a direct summand $N$ of $M$ such that $N \subseteq \operatorname{Im}(\gamma)$. We have $\frac{\operatorname{Im}(\gamma)}{N}=\frac{N \oplus N^{\prime}}{N} \subseteq$ $\frac{N+\operatorname{Rad}(M)}{N} \subseteq \operatorname{Rad}\left(\frac{M}{N}\right)$.

Definition 2.2. A module $M$ has the property $\left(N-G P^{*}\right)$ if, for every homomorphism $\gamma: M \longrightarrow N$, there exists a direct summand $L$ of $N$ such that $L \subseteq \operatorname{Im}(\gamma)$ and $\frac{\operatorname{Im} \gamma}{L} \subseteq \operatorname{Rad}\left(\frac{N}{L}\right)$.

It is clear that a right module $M$ has the property $\left(G P^{*}\right)$ if and only if $M$ has the property $\left(M-G P^{*}\right)$.

Recall from $[4,3.6]$ that a submodule $N$ of $M$ is called coclosed in $M$ if, $N$ has no proper submodule $K$ for which $K \subset N$ is cosmall in $M$, that is, $\frac{N}{K} \ll \frac{M}{K}$. Obviously any direct summand $N$ of $M$ is coclosed in $M$. 
Theorem 2.1. Let $M$ and $N$ be right $R$-modules. Then $M$ has the property $\left(N-G P^{*}\right)$ if and only if, for all direct summands $M^{\prime}$ and a coclosed submodule $N^{\prime}$ of $N, M^{\prime}$ has the property $\left(N^{\prime}-G P^{*}\right)$.

Proof. ( $\Longrightarrow)$ Let $M^{\prime}=e M$ for some $e^{2}=e \in \operatorname{End}_{R}(M)$ and let $N^{\prime}$ be a coclosed submodule of $N$. Assume that $\alpha \in \operatorname{Hom}\left(M^{\prime}, N^{\prime}\right)$. Since $\alpha(e M)=$ $\alpha\left(M^{\prime}\right) \subseteq N^{\prime} \subseteq N$ and $M$ has the property $\left(N-G P^{*}\right)$, there exists a decomposition $N=N_{1} \oplus N_{2}$ such that $N_{1} \subseteq \operatorname{Im}(\alpha(e))$ and $N_{2} \cap \operatorname{Im}(\alpha(e)) \subseteq$ $\operatorname{Rad}\left(M_{2}\right) \subseteq \operatorname{Rad}(N)$. Then we have $N^{\prime}=N_{1} \oplus\left(N_{2} \cap N^{\prime}\right)$ by the modular law. Since $N^{\prime}$ is a coclosed submodule of $N$, then $\operatorname{Rad}\left(N^{\prime}\right)=\operatorname{Rad}(N) \cap N^{\prime}$ by [4, 3.7(3)]. So $N_{2} \cap N^{\prime} \cap \operatorname{Im}(\alpha) \subseteq \operatorname{Rad}\left(N^{\prime}\right)$. By using [4, 3.7(3)] once again, we get $N_{2} \cap N^{\prime} \cap \operatorname{Im}(\alpha) \subseteq \operatorname{Rad}\left(N_{2} \cap N^{\prime}\right)$. Therefore $M^{\prime}$ has the property $\left(N^{\prime}-G P^{*}\right)$.

$(\Longleftarrow)$ Clear.

Corollary 2.1. The following conditions are equivalent for a module $M$.

(1) $M$ has the property $\left(G P^{*}\right)$.

(2) For any coclosed submodule $N$ of $M$, every direct summand $L$ of $M$ has the property $\left(N-G P^{*}\right)$.

Corollary 2.2. Every direct summand of a module that have the property $\left(G P^{*}\right)$ has the property $\left(G P^{*}\right)$.

Proposition 2.2. Let $M$ be an indecomposable module. Assume that, for $\delta \in \operatorname{End}_{R}(M), \operatorname{Im}(\delta) \subseteq \operatorname{Rad}(M)$ implies $\delta=0$. Then, $M$ has the property $\left(G P^{*}\right)$ if and only if every nonzero endomorphism $\delta \in \operatorname{End}_{R}(M)$ is an epimorphism.

Proof. Assume that $0 \neq \delta \in \operatorname{End}_{R}(M)$. Since $M$ has the property $\left(G P^{*}\right)$, there exists a decomposition $M=M_{1} \oplus M_{2}$ with $M_{1} \subseteq \operatorname{Im}(\delta)$ and $M_{2} \cap$ $\operatorname{Im}(\delta) \subseteq \operatorname{Rad}\left(M_{2}\right)$. Since $M$ is indecomposable, $M_{1}=0$ or $M_{1}=M$. If $M_{1}=0$, then $\operatorname{Im}(\delta) \subseteq \operatorname{Rad}(M)$. By the hypothesis $\delta=0$; a contradiction. Thus, $M_{1}=M$ and hence, $\delta$ is epimorphism. The converse is clear.

Recall from $[4,4.27]$ that a module $M$ is said to be Hopfian if every surjective endomorphism of $M$ is an isomorphism.

Proposition 2.3. Let $M$ be a noetherian module that has the property $\left(G P^{*}\right)$. If every endomorphism $\gamma$ of $M, \operatorname{Im}(\gamma) \subseteq \operatorname{Rad}(M)$ implies that $\gamma=0$. Then there exists a decomposition $M=M_{1} \oplus M_{2} \oplus \ldots \oplus M_{n}$, where $M_{i}$ is an indecomposable noetherian modules that has the property $\left(G P^{*}\right)$ for which $\operatorname{End}_{R}\left(M_{i}\right)$ is a division ring.

Proof. Since $M$ is noetherian, it has a finite decomposition noetherian direct summands. By Corollary 2.2, every direct summand has the property $\left(G P^{*}\right)$. By Proposition 2.2, in view of the fact that every noetherian module is Hopfian, each indecomposable direct summand has a division ring. 
Definition 2.3. A module $M$ is called $G^{*}$-supplemented if, for every $\gamma \in$ $\operatorname{End}_{R}(M), \operatorname{Im}(\gamma)$ has a $\operatorname{Rad}$-supplement in $M$, and a module $M$ is called amply $G^{*}$-supplemented if, for every $\gamma \in \operatorname{End}_{R}(M), \operatorname{Im}(\gamma)$ has ample $\operatorname{Rad}$ supplements in $M$.

It is clear that every module that has the property $\left(G P^{*}\right)$ is $G^{*}$-supplemented by the Definition 2.3.

Proposition 2.4. Let $M$ be an amply $G^{*}$-supplemented $R$-module. Then every direct summand of $M$ is amply $G^{*}$-supplemented.

Proof. Let $N$ be a direct summand of $M$. Then $M=N \oplus N^{\prime}$ for some $N^{\prime} \subseteq M$. Suppose that $f \in \operatorname{End}_{R}(N)$ and $N=\operatorname{Im}(f)+K$. Thus, $M=$ $\operatorname{Im}(f)+K+N^{\prime}$. Note that $\operatorname{Im}(f)=\operatorname{Im}(\iota f \pi)$, where $\iota$ is the injection map from $N$ to $M$ and $\pi$ is the projection map from $M$ onto $N$. Since $M$ is amply $G^{*}$-supplemented, there exists a Rad-supplement $L$ of $N^{\prime}+K$ with $L \subseteq \operatorname{Im}(f)$. We get $K \cap L \subseteq\left(N^{\prime}+K\right) \cap L \subseteq \operatorname{Rad}(L)$ and $M=L+N^{\prime}+K$. Thus $N=K+L$ by the modular law. So $K+L=N$ and $K \cap L \subseteq \operatorname{Rad}(L)$. Therefore $N$ is amply $G^{*}$-supplemented.

Proposition 2.5. Let $M$ be an amply $G^{*}$-supplemented distributive module and let $N$ be a direct summand of $M$ for every Rad-supplement submodule $N$ of $M$. Then $M$ is a $G^{*}$-supplemented module.

Proof. Let $f \in \operatorname{End}_{R}(M)$, let $K$ be a $R a d$-supplement of $\operatorname{Im}(f)$ in $M$, and let $N$ a Rad-supplement of $K$ in $M$ with $N \subseteq \operatorname{Im}(f)$. By the hypothesis, $M=N \oplus N^{\prime}$ for some $N^{\prime} \leq M . \quad \operatorname{Im}(f)=\operatorname{Im}(f) \cap(N+K)=N+$ $(\operatorname{Im}(f) \cap K)$. Since $\operatorname{Im}(f) \cap K \subseteq \operatorname{Rad}(K)$, then we have $\operatorname{Im}(f) \cap K \cap N^{\prime} \subseteq$ $\operatorname{Rad}(K)$. As $M$ is distributive, $\operatorname{Im}(f)+K \cap N^{\prime}=N+K=M$ and $K=K \cap\left(N \oplus N^{\prime}\right)=(K \cap N) \oplus\left(K \cap N^{\prime}\right)$. So $K \cap N^{\prime}$ is a direct summand of $K$. Since $\operatorname{Im}(f) \cap K \cap N^{\prime} \subseteq K \cap N^{\prime}, \operatorname{Im}(f) \cap K \cap N^{\prime} \subseteq \operatorname{Rad}\left(K \cap N^{\prime}\right)$. Therefore $M$ is $G^{*}$-supplemented.

Definition 2.4. A module $M$ is called $N-G^{*}$-supplemented if, for every homomorphism $\phi: M \longrightarrow N$, there exists $L \leq N$ such that $\operatorname{Im}(\phi)+$ $L=N$ and $\operatorname{Im}(\phi) \cap L \subseteq \operatorname{Rad}(L)$. It is clear that the right module $M$ is $G^{*}$-supplemented if and only if $M$ is $M-G^{*}$-supplemented.

Recall from [11] that a submodule $U$ of an $R$-module $M$ is called fully invariant if $f(U)$ is contained in $U$ for every $R$-endomorphism $f$ of $M$. A module $M$ is called $d u o$, if for every submodule of $M$ is fully invariant [9].

Theorem 2.2. Let $M_{1}, M_{2}$ and $N$ be modules. If $N$ is $M_{i}-G^{*}-$ supplemented for $i=1,2$, then $N$ is $M_{1} \oplus M_{2}-G^{*}$-supplemented. The converse is true if $M_{1} \oplus M_{2}$ is a duo module.

Proof. Suppose that $N$ is $M_{i}-G^{*}$-supplemented for $i=1,2$. We prove that $N$ is $M_{1} \oplus M_{2}-G^{*}$-supplemented. Let $\phi=\left(\pi_{1} \phi, \pi_{2} \phi\right)$ be any homomorphism from $N$ to $M_{1} \oplus M_{2}$, where $\pi_{i}$ is the projection map from $M_{1} \oplus M_{2}$ 
into $M_{i}$ for $i=1,2$. Since $N$ is $M_{i}-G^{*}$-supplemented, there exists a submodule $K_{i}$ of $M_{i}$ such that $\pi_{i} \phi N+K_{i}=M_{i}$ and $\pi_{i} \phi N \cap K_{i} \subseteq \operatorname{Rad}\left(K_{i}\right)$ for $i=1,2$. Let $K=K_{1} \oplus K_{2}$. Then $M_{1} \oplus M_{2}=\pi_{1} \phi N+\pi_{2} \phi N+K_{1}+K_{2}=$ $\phi N+K$. Since $\phi N \cap\left(K_{1}+K_{2}\right) \subseteq\left(\phi N+K_{1}\right) \cap K_{2}+\left(\phi N+K_{2}\right) \cap K_{1}$, we get $\phi N \cap\left(K_{1}+K_{2}\right) \subseteq\left(\phi N+M_{1}\right) \cap K_{2}+\left(\phi N+M_{2}\right) \cap K_{1}$. Since $\phi N+M_{1}=\pi_{2} \phi N \oplus M_{1}$ and $\phi N+M_{2}=\pi_{1} \phi N \oplus M_{2}$, we conclude that $\phi N \cap K \subseteq\left(\pi_{2} \phi N \cap K_{2}\right)+\left(\pi_{1} \phi N+K_{1}\right)$. Since $\pi_{i} \phi N \cap K_{i} \subseteq \operatorname{Rad}\left(K_{i}\right)$ for $i=$ 1,2 , we get $\phi N \cap K \subseteq \operatorname{Rad}(K)$. Hence, $N$ is $M_{1} \oplus M_{2}-G^{*}$-supplemented.

Conversely, let $N$ be $M_{1} \oplus M_{2}-G^{*}$-supplemented. Let $\phi$ be a homomorphism from $N$ to $M_{1}$. Then $\operatorname{Im}(\iota \phi)=\operatorname{Im}(\phi)$, where $\iota$ is the canonical inclusion from $M_{1}$ to $M_{1} \oplus M_{2}$. Since $N$ is $M_{1} \oplus M_{2}-G^{*}$-supplemented, there exists $K \subseteq M_{1} \oplus M_{2}$ such that $M_{1} \oplus M_{2}=\operatorname{Im}(\phi)+K$ and $\operatorname{Im}(\phi) \cap K \subseteq$ $\operatorname{Rad}(K)$. Thus, $M_{1}=\operatorname{Im}(\phi)+\left(K \cap M_{1}\right)$ and $\operatorname{Im}(\phi) \cap K \cap M_{1}=\operatorname{Im}(\phi) \cap K \subseteq$ $\operatorname{Rad}(K)$. As $M_{1} \oplus M_{2}$ is a duo module and $K=K_{1} \oplus K_{2} \leq M_{1} \oplus M_{2}, K \cap M_{1}$ is a direct summand of $K$. Hence $\operatorname{Im}(\phi) \cap K \cap M \subseteq \operatorname{Rad}\left(K \cap M_{1}\right)$. Therefore $N$ is an $M_{1}-G^{*}$-supplemented.

Corollary 2.3. Suppose that $M=M_{1} \oplus M_{2}$ and $M$ is a $G^{*}$-supplemented module for $i=1,2$. Then $M$ is $G^{*}$-supplemented and, for every $f \in$ $\operatorname{End}_{R}(M), \operatorname{Im}(f)$ has a Rad-supplement of the form $K_{1}+K_{2}$ with $K_{1} \subseteq M_{1}$ and $K_{2} \subseteq M_{2}$.

Proof. Follows from the proof of Theorem 2.2.

Theorem 2.3. Let $M=\oplus_{i=1}^{n} M_{i}$ be a module and $M_{i}$ be a fully invariant submodule of $M$ for all $i \in\{1,2, \ldots, n\}$. Then $M$ has the property $\left(G P^{*}\right)$ if and only if $M_{i}$ has the property $\left(G P^{*}\right)$ for all $i \in\{1,2, \ldots, n\}$.

Proof. The necessity follows from Theorem 2.1. Conversely, let $N_{i}$ be a module that have the property $\left(G P^{*}\right)$ for all $i \in\{1,2, \ldots, n\}$. Also let $\phi=\left(\phi_{i j}\right)_{i, j \in\{1,2, \ldots, n\}} \in \operatorname{End}(M)$ be arbitrary, where $\left(\phi_{i j}\right) \in \operatorname{Hom}\left(M_{j}, M_{i}\right)$. Since $M_{i}$ is a fully invariant submodule of $M$ for all $i \in\{1,2, \ldots, n\}$, we get $\operatorname{Im}(\phi)=\oplus_{i=1}^{n} \operatorname{Im}\left(\phi_{i i}\right)$. As $M_{i}$ has the property $\left(G P^{*}\right)$, there exists a direct summand $N_{i}$ of $M_{i}$ and a submodule $K_{i}$ of $M_{i}$ with $N_{i} \subseteq \operatorname{Im}\left(\phi_{i i}\right), \operatorname{Im}\left(\phi_{i i}\right)=$ $N_{i}+K_{i}$ and $K_{i} \subseteq \operatorname{Rad}\left(M_{i}\right)$. We say $N=\oplus_{i=1}^{n} N_{i}$. Then $N$ is a direct summand of $M$. Moreover, $\operatorname{Im}(\phi)=\oplus_{i=1}^{n} \operatorname{Im}\left(\phi_{i i}\right)=\sum_{i=1}^{n} N_{i}+\sum_{i=1}^{n} K_{i}$ and $\oplus_{i=1}^{n} K_{i} \subseteq \operatorname{Rad}\left(\oplus_{i=1}^{n} M_{i}\right)=\operatorname{Rad}(M)$. Therefore $M$ has the property $\left(G P^{*}\right)$.

Theorem 2.4. The following assertions are equivalent for a ring $R$.

(1) $R$ is generalized $f$-semiperfect.

(2) $R_{R}$ is f-Rad-supplemented.

(3) Every cyclic right ideal has a Rad-supplement in $R_{R}$.

(4) $R_{R}$ is a $G^{*}$-supplemented module.

(5) $R_{R}$ has the property $\left(G P^{*}\right)$.

Proof. (1) $\Leftrightarrow(2) \Leftrightarrow(3)$ By [8, Theorem 2.22]. 
$(3) \Rightarrow(4)$ is clear because $\operatorname{Im}(\gamma)$ is cyclic for every $\gamma \in \operatorname{End}_{R}\left(R_{R}\right)$.

(4) $\Rightarrow(3)$ Assume that $I=a R$ is any cyclic right ideal of $R$. Consider the $R$-homomorphism $\phi: R_{R} \longrightarrow R_{R}$ defined by $\phi(r)=a r$; where $r \in R$. Then $\operatorname{Im}(\phi)=I$. By $(4), \operatorname{Im}(\phi)=I$ has a $R a d$-supplement in $R_{R}$.

$(5) \Rightarrow(4)$ is clear.

$(5) \Rightarrow(3)$ Suppose that $R_{R}$ has the property $\left(G P^{*}\right)$. Let $J=b R$ is any cyclic right ideal of $R$. Consider the $R$-homomorphism $\phi: R_{R} \longrightarrow R_{R}$ defined by $\phi(r)=b r$; where $r \in R$. Then $\operatorname{Im}(\phi)=J$. By (5), there exists submodules $R_{1}, R_{2}$ of $R_{R}$ such that $R_{R}=R_{1} \oplus R_{2}, R_{1} \subseteq \operatorname{Im}(\phi)=J$ and $R_{2} \cap \operatorname{Im}(\phi) \subseteq \operatorname{Rad}\left(R_{2}\right)$. So $R_{R}=J+R_{2}$ and $J \cap R_{2} \subseteq \operatorname{Rad}\left(R_{2}\right)$. Thus $R_{2}$ is a $R a d$-supplement of $J$ in $R_{R}$.

The equivalent condition for the property $\left(P^{*}\right)$ if every submodule $N$ of $M$ there exist submodules $K, K$ of $M$ such that $K \leq N, M=K \oplus K^{\prime}$ and $N \cap K^{\prime} \subseteq \operatorname{Rad}\left(K^{\prime}\right)$ (See [1]).

Proposition 2.6. Let $M$ be a module which has the property $\left(P^{*}\right)$. Then $M$ has the property $\left(G P^{*}\right)$.

Proof. Let $\phi: M \longrightarrow M$ be any homomorphism. Since $M$ has the property $\left(P^{*}\right)$, there exist submodules $K, K$ of $M$ such that $K \leq \operatorname{Im}(\phi), M=K \oplus K^{\prime}$ and $\operatorname{Im}(\phi) \cap K^{\prime} \subseteq \operatorname{Rad}\left(K^{\prime}\right)$. So $M$ has the property $\left(G P^{*}\right)$.

Example 2.1. (See [2]) Let $F$ be any field. Consider the commutative ring $R$ which is the direct product $\prod_{i=0}^{\infty} F_{i}$, where $F_{i}=F$. So $R_{R}$ is a regular ring which is not semisimple. The right $R$-module $R$ is f-Rad-supplemented but not $R a d$-supplemented. Since $R_{R}$ is f-Rad-supplemented, $R_{R}$ has the property $\left(G P^{*}\right)$ by Theorem 2.4. As $R_{R}$ is not $R a d$-supplemented, $R_{R}$ has not the property $\left(P^{*}\right)$.

\section{REFERENCES}

[1] I. Al-Khazzi, P. F. Smith, Modules with Chain Conditions on Superfluous Submodules, Algebra Discrete Math. J., Vol. 3 (2006) , pp. 1-16.

[2] F.W. Anderson, K. R. Fuller, Rings and Categories of Modules, Springer Verlag, Berlin-Heidelberg-New York, 1988.

[3] E. Büyükaşı, E. Mermut, S. Özdemir, Rad-supplemented Modules, Rend. Sem. Mat. Univ. Padova, Vol. 124, (2010), pp. 157-177.

[4] J. Clark, C. Lomp,N. Vanaja, R. Wisbauer Lifting Modules. Supplements and Projectivity in Module Theory, Frontiers in Mathematics, Birkhäuser, 2006.

[5] H. Çalışıcı, E. Türkmen, Generalized $\oplus$-supplemented modules, Algebra Discrete Math., Vol. 10, No.2 (2010), pp.10-18.

[6] S. H. Mohamed, B. J. Müller, Continuous and discrete modules, London Math. Soc. LNS 147 Cambridge University, Cambridge, 1990. 
[7] B. Nişancı Türkmen, A. Pancar, A Generalization of Rad-supplemented Modules, International Journal of Pure and Applied Mathematics, Vol. 68, No. 4 (2011), pp. $477-485$.

[8] B. Nişancı Türkmen, A. Pancar, Generalized f-semiperfect Modules, Communications in Mathematics and Applications, Vol. 4, No. 1 (2013) , pp. 85-92.

[9] A. Ç. Özcan, A. Harmacı, P. F. Smith, Duo modules, Glasgow Math. J., Vol. 48 (2006) , pp. 533-545.

[10] Y. Wang, N. Ding, Generalized Supplemented Modules, Taiwanese J. Math., Vol. 6 (2006), pp. 1589-1601.

[11] R. Wisbauer, Foundations of Modules and Rings, Gordon and Breach, 1991.

[12] W. Xue, Characterizations of semiperfect and perfect modules, Publicacions Matematiques, Vol. 40, No. 1 (1996), pp. 115-125.

[13] H. Zöschinger, Supplemented Modules Over Dedekind Rings, J. Algebra, Vol. 29 (1974) , pp. 42-56.

\author{
Burcu Nişanci Türkmen \\ Amasya University \\ Faculty of Art and Science \\ Deparment of Mathematics \\ 05100 AMASYA \\ TURKEY \\ E-mail address: burcunisancie@hotmail.com
}

